\title{
Interleave Division Multiplexing Aided Space-Time Coding for High-Throughput Uplink Cooperative Communications
}

\author{
Rong Zhang and Lajos Hanzo \\ School of ECS., Univ. of Southampton, SO17 1BJ, UK. \\ Tel: +44-23-80-593 125, Fax: +44-23-80-593045 \\ Email: lh@ecs.soton.ac.uk, http://www-mobile.ecs.soton.ac.uk
}

\begin{abstract}
In this paper, we design and investigate a novel Interleave Division Multiplexing based Space-Time Code (IDM-STC) in the context of cooperative communications. We outline the particular signalling scheme used for exchanging the necessary information amongst the cooperating MSs and suggest an efficient interleaver allocation scheme, which is capable of uniquely and unambiguously differentiating the different MSs' signals with the aid of their user and antenna-specific interleavers. We then characterize the achievable performance of our proposed IDM-STC design and compare it to that of the traditional $G_{2}$ and $G_{4}$ Space-Time Block Code (STBC) invoked for cooperative communications. Our cooperative IDM-STC scheme is flexible in terms of forming a cluster of cooperative users, it is power-efficient and capable of maintaining a high rate, in particular when combined with non-uniform power allocation.
\end{abstract}

\section{INTRODUCTION}

Multiple Input Multiple Output (MIMO) systems [1] are capable of providing both diversity and coding gains in the context of SpaceTime Codes (STC) [2] as well as of supporting a high multiplexing gain, when using for example Bell-Labs Layered Space Time Architecture (BLAST) [3]. However, at the Mobile Station (MS), it may be impractical to accommodate multiple antennas. Alternatively, the novel concept of cooperative communications allows us to assign the MIMO elements to geographically separated cooperating MSs, which are no longer prone to shadowing-induced correlated fading, leading to the concept of Virtual MIMOs (VMIMO) [4], [5].

Hence STC based VMIMO designs [6] are attractive for employment in cooperative communications, where the cooperating MSs' independently fading signals jointly constitute a STC codeword. Recently, an Interleave Division Multiplexing Space-Time Code (IDMSTC) by $\mathrm{Wu}$ and Ping was proposed in [7], where its potential applicability in cooperative communications was also alluded to. The resultant IDM-STC was then investigated and analyzed in [8], where a similar performance was reported to that attained by Alamouti's STBC. This motivated us to design and investigate the proposed IDMSTC in the context of cooperative communications.

Our design of IDM-STC was specifically contrived for cooperative communications by appropriately adopting the Multilayer IDM-STC concept [7], where we treat each cooperating MS as an IDMSTC layer. Instead of using multilevel modulation schemes [9], we employ sigma mapping for creating an error-resilient binary cooperative system. We also design a realistic signalling scheme required for exchanging the information amongst the cooperating MSs and contrast the benefits of IDM-STCs to those of the traditional $G_{2}$ and $G_{4}$ Space-Time Block Code (STBC) design [10]. More specifically, the novel contribution of this paper is that we design an error-resilient, yet high-throughput IDM-STC scheme suitable for

Acknowledgments: The work reported in this paper has formed part of the Core 4 Research Programme of the Virtual Center of Excellence in Mobile and Personal Communications, Mobile VCE, www.mobilevce.com, whose funding support, including that of EPSRC, is gratefully acknowledged. Fully detailed technical reports on this research are available to Industrial Members of Mobile VCE. cooperative communications and characterize its achievable rate, power-efficiency and flexibility.

The rest of the paper is organized as follows. In Section II, we describe the cooperative scenarios considered and introduce the IDMSTC transceiver architecture designed for cooperative communications. In Section III, we design a practical signalling scheme for exchanging the necessary information amongst the cooperating MSs and suggest an efficient interleaver allocation scheme. In Section IV, we outline the achievable benefits compared to the traditional $G_{2}$ and $G_{4}$ STBC design. Finally, we conclude our discourse in Section V.

\section{System ARChitecture}

\section{A. Cooperative Scenarios}

Consider a cluster of uplink transmitters cooperatively communicating with a destination Base Station (BS) employing a single receive antenna. Similarly, each cooperating transmitter has a single transmit antenna, resulting in a Virtual Multiple Input Single Output (VMISO) system. We define two modes of operation for a cooperating MS of a cluster, namely 1) active, when the MS is conveying both its own information and other cooperating MSs' information; 2) relaying, when the MS is available for conveying other MSs' information.

We assume that the channels amongst the cooperating MSs are "ideal", which assumes that the information exchanged amongst the cooperating MSs is error-free and perfectly synchronized. We assume furthermore that the channels between the cooperating MSs and the BS exhibit independent identically distributed (i.i.d) Rayleigh fading and that the Channel State Information (CSI) is perfectly known both at the BS's and the Relay Station's (RS) receiver. ${ }^{1}$

\section{B. Cooperative Transmitter}

In this VMISO system, we assume having a total of $N$ transmit antennas, $K$ cooperating MSs and $(N-K)$ RSs in a cluster, where we have $N \geq K$, as seen in Fig. 1 .

The $k$ th MS's transmitted bit stream $b_{k}$ is firstly channel encoded by $C_{1}$ at a rate of $r_{1}$, yielding the encoded stream $c_{k}^{1}$. The resultant channel encoded stream is randomly interleaved by a user-specific chip-interleaver $\pi_{k}^{u}$, resulting in $\hat{c}_{k}^{1}$. This stream is then repetition coded by $C_{2}$ at a rate of $r_{2}$, resulting in $c_{k}^{2}$, which is then $\mathrm{S} / \mathrm{P}$ converted to $N$ parallel streams and mapped to the $N$ antennas, yielding the information $c_{k, n}^{2}$ of MS $k$ at antenna $n$. Then each stream $c_{k, n}^{2}$, which the MS intends to transmit with the aid of its $n$th cooperating partner, is again randomly interleaved by an antennaspecific chip-interleaver $\pi_{n}^{a}$, yielding $\hat{c}_{k, n}^{2}$, before sending it to the multilayer mapping stage, as seen in Fig. 1.

\footnotetext{
${ }^{1}$ Naturally, this is a very demanding assumption, since estimating all the related channels imposes a high complexity. As a first step towards eliminating this demanding assumption, we could consider differentially encoded and noncoherently detected schemes.
} 


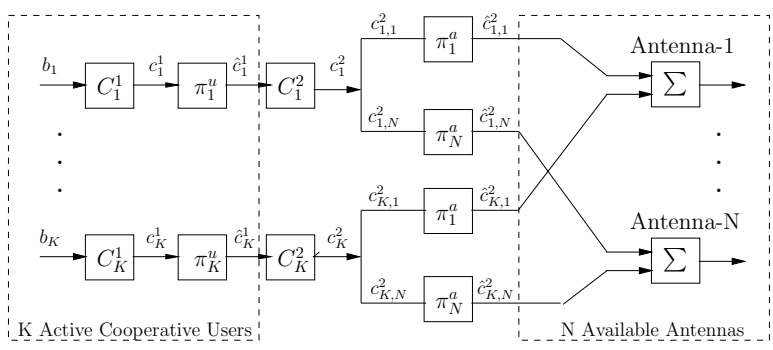

Fig. 1. The $K$-user cooperative IDM-STC UpLink transmitter

1) IDM-STC: In this paper, we employ repetition codes of coderate $r_{1}$ and $r_{2}$ for both $C^{1}$ and $C^{2}$, respectively, resulting in a total code-rate of $R=\left(r_{1} \times r_{2}\right)$. When considering a cluster of $N$ available antennas, the overall rate of the IDM-STC scheme becomes:

$$
r_{I D M}=N \times R,
$$

which is always less than unity, when we assume $N \leq 1 / R$.

We will further consider three typical settings of $r_{1}$ and $r_{2}$ given a fixed total code-rate $R$, namely: 1) $r_{1}=R$ and $r_{2}=1$, i.e. when we employ the repetition code $C^{1}$ only. The interleaver $\pi_{k}^{u}$ and S/P conversion of Fig. 1 ensures that the encoded bits $c_{k}^{1}$ are randomly dispersed across both the spatial and the time domain. 2) $r_{1}=1$ and $r_{2}=R$, i.e. only the repetition code $C^{2}$ is employed and the following S/P conversion of Fig. 1 ensures that the encoded bits $c_{k}^{2}$ are directly mapped to each antenna, since the interleaver $\pi_{n}^{a}$ of Fig. 1 operates after S/P conversion. 3) $r_{1}=R N$ and $r_{2}=1 / N$.

2) Multilayer mapping: In contrast to classic mapping and modulation schemes, such as PSK and QAM, sigma mapping [11], which are based on the theory of multiuser communications, was designed to generate an approximately Gaussian distributed transmitted signal, which allows the system to approach the Shannon capacity.

Let us hence consider the multilayer mapping of $K$ MSs' cooperative bit streams, i.e. $\hat{c}_{k, n}^{2}, \forall k$ at the $n$th antenna. Let us assume that we want to transmit $m_{k, n}$ bits/symbol with the aid of each substream $\hat{c}_{k, n}^{2}$, where the resultant symbol vector is denoted by $\mathbf{v}_{k, n}=\left[\hat{c}_{k, n, 1}^{2}, \cdots, \hat{c}_{k, n, m_{k, n}}^{2}\right]$. Then the total number of bits/symbol transmitted by all the $K$ MSs that are mapped to the $n$th antenna, $n=1, \cdots, N$ is given by:

$$
\mathcal{L}_{n}=\sum_{k=1}^{K} m_{k, n} .
$$

We refer the number of bits as the number of layers. The supersymbol vector $\mathbf{s}_{n}=\left[\mathbf{v}_{1, n}, \cdots, \mathbf{v}_{K, n}\right]$ hosting all the $K$ symbol vectors is then weighted by the $n$th antenna's coefficient $\mathbf{u}_{n}$ and superimposed to generate the $K$-MS "super-symbol" in the form of

$$
\mathbf{x}_{n}=\mathbf{u}_{n} \mathbf{s}_{n}^{T},
$$

where $(\cdot)^{T}$ denotes the transpose and the weighting coefficient vector is given by:

$$
\mathbf{u}_{n}=\left[\rho_{n, 1} e^{j \theta_{n, 1}}, \cdots, \rho_{n, \mathcal{L}_{n}} e^{j \theta_{n, \mathcal{L}_{n}}}\right],
$$

with the entries of $\rho_{n, m}$ and $\theta_{n, m} \in[0, \pi]$ representing the layerspecific amplitude ${ }^{2}$ and phase $^{3}$ of the $n$th antenna's stream, respec-

\footnotetext{
${ }^{2}$ The rationale of allocating a different power $\rho_{n, m}$ to each of the $\mathcal{L}_{n}$ layers is philosophically similar to that of the multilevel coding concept, where we create a number of different protection levels and detect them by gleaning extrinsic information from the previously decoded levels using multistage decoding.

${ }^{3}$ The associated phase rotation has two benefits, namely that of 1) reducing the Peak-to-Average Power Ratio (PAPR) of the transmitted "super-symbol" $\mathbf{x}_{n} ; 2$ ) making the "super-symbol" having $\mathcal{L}_{n}$ layers more distinguishable for the detector.
}

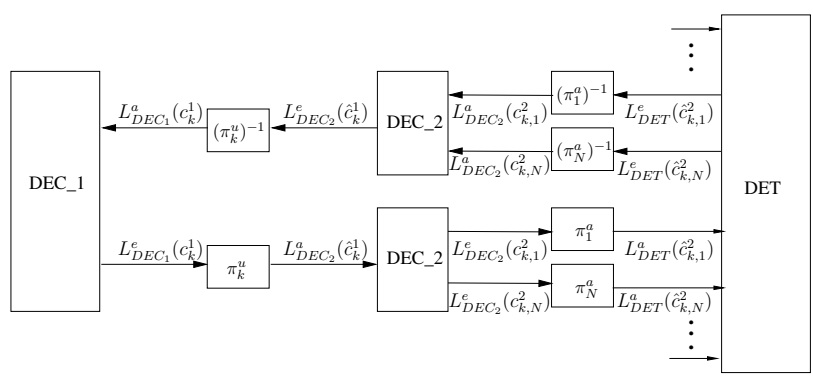

Fig. 2. The iterative IDM-STC UpLink receiver of the $K$ users

tively.

We assume that the number of layers $\mathcal{L}_{n}$ and the weighting coefficient vector $\mathbf{u}_{n}$ is the same for all of the $N$ antennas, which implies that we have $\mathcal{L}_{n}=\mathcal{L}, \forall n$ and $\mathbf{u}_{n}=\mathbf{u}, \forall n$. Furthermore, we employ a layer-specific uniform phase rotation so that the $\mathcal{L}_{n}$ number of layers are uniformly phase-rotated on the two-dimensional signal space.

\section{Turbo Receiver of IDM-STC}

The discrete-time received signal $y$ is given by:

$$
\begin{aligned}
y & =\mathbf{h} \mathbf{x}+\nu, \\
& =\mathbf{h U s}+\nu,
\end{aligned}
$$

where we have:

$$
\begin{aligned}
\mathbf{h} & =\left[h_{1}, \cdots, h_{N}\right]_{1 \times N}, \\
\mathbf{s} & =\left[\mathbf{s}_{1}, \cdots, \mathbf{s}_{N}\right]_{N \mathcal{L} \times 1}^{T}, \\
\mathbf{U} & =\operatorname{diag}\left[\mathbf{u}_{1}, \cdots, \mathbf{u}_{N}\right]_{N \times N \mathcal{L}},
\end{aligned}
$$

where $\mathbf{h}$ denotes the i.i.d Channel Impulse Response (CIR) vector of the VMISO system. Each entry $\mathbf{u}_{n}=\mathbf{u}$ in $\mathbf{U}$ can be viewed as a complex-valued scaling vector of the transmitted binary signal vector $\mathbf{s}_{n}$ corresponding to the $n$th antenna imposing an amplitude scaling and phase rotation action. Finally, $\nu \sim \mathcal{N}_{c}\left(0, \sigma_{n}^{2}\right)$ in Eq. (5) is a complex-valued Additive White Gaussian Noise (AWGN) process having $\sigma_{n}^{2}=N_{0} / 2$ per dimension. We rewrite Eq. (5) as:

$$
y=\mathbf{H s}+\nu,
$$

where $\mathbf{H}=\mathbf{h} \mathbf{U}$ is the equivalent CIR matrix. Hence, by using sigma mapping, a binary system is constructed, which facilitates a realvalued processing at the receiver side.

The turbo receiver consists of a Soft In Soft Out (SISO) Detector (DET) and a bank of $K$ individual SISO decoders (DEC), as seen in Fig. 2. The SISO DET employs the low complexity Soft Interference Cancellation (SoIC) scheme of [12].

Let us now consider the $i$ th bit $s_{i}$ of the transmitted super-symbol vector $\mathbf{s}$. Then Eq. (6) can be written as

$$
y=H_{i} s_{i}+\xi,
$$

where $\xi=\sum_{j \neq i} H_{j} s_{j}+\nu$ represents the interference plus noise. In a binary system the real part $(R e)$ of $H_{i}^{*} y$ constitutes sufficient statistics for estimating $s_{i}$, where $(\cdot)^{*}$ denotes the conjugate, resulting in:

$$
\operatorname{Re}\left(H_{i}^{*} y\right)=\left|H_{i}\right|^{2} s_{i}+\operatorname{Re}\left(H_{i}^{*} \xi\right) .
$$

We denote the soft estimate of a variable $a$ by $(\hat{a})$. Then, the soft estimate $\operatorname{Re}\left(H_{i}^{*} \hat{\xi}\right)$ and its variance $\mathbf{V}\left[\operatorname{Re}\left(H_{i}^{*} \hat{\xi}\right)\right]$ are given by:

$$
\begin{aligned}
\operatorname{Re}\left(H_{i}^{*} \hat{\xi}\right)= & H_{i}^{R e} \hat{y}^{R e}+H_{i}^{I m} \hat{y}^{I m}-\left|H_{i}\right|^{2} \hat{s}_{i}, \\
\mathbf{V}\left[\operatorname{Re}\left(H_{i}^{*} \hat{\xi}\right)\right]= & \left(H_{i}^{R e}\right)^{2} \mathbf{V}\left(\hat{y}^{R e}\right)+\left(H_{i}^{I m}\right)^{2} \mathbf{V}\left(\hat{y}^{I m}\right) \\
& -\left|H_{i}\right|^{4} \mathbf{V}\left(\hat{s}_{i}\right)+2 H_{i}^{R e} H_{i}^{I m} \phi,
\end{aligned}
$$



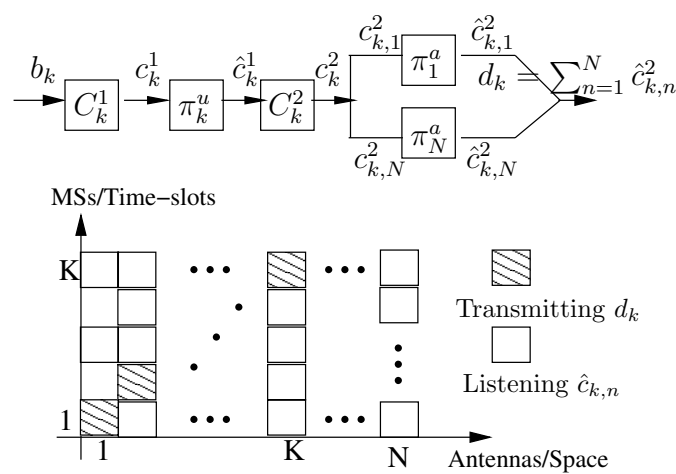

Fig. 3. The Phase-I cooperation of the $K$-user UpLink IDM-STC

where we have $\phi=\sum_{i=1}^{N \mathcal{L}} H_{i}^{R e} H_{i}^{I m} \mathbf{V}\left(\hat{s}_{i}\right)$ and $\operatorname{Im}(\cdot)$ represents the imaginary part of a complex number. The soft estimate $\hat{y}^{R e}$ and its variance $\mathbf{V}\left(\hat{y}^{R e}\right)$ may be expressed as:

$$
\begin{aligned}
\hat{y}^{R e} & =\sum_{i=1}^{N \mathcal{L}} H_{i}^{R e} \hat{s}_{i}, \\
\mathbf{V}\left(\hat{y}^{R e}\right) & =\sum_{i=1}^{N \mathcal{L}}\left(H_{i}^{R e}\right)^{2} \mathbf{V}\left(\hat{s}_{i}\right)+\sigma_{n}^{2} .
\end{aligned}
$$

We remark that Eq. (11) and Eq. (12) also hold for the imaginary counterpart. The soft estimate $\hat{s}_{i}$ can be represented as $\hat{s}_{i}=$ $\tanh \left(L_{D E T}^{a}\left(s_{i}\right) / 2\right)$, while its variance is given by $\mathbf{V}\left(\hat{s}_{i}\right)=1-\hat{s}_{i}^{2}$. Thus, the extrinsic information $L_{D E T}^{e}\left(s_{i}\right)$ is given by:

$$
L_{D E T}^{e}\left(s_{i}\right)=2\left|H_{i}\right|^{2} \frac{\operatorname{Re}\left(H_{i}^{*} y\right)-\operatorname{Re}\left(H_{i}^{*} \hat{\xi}\right)}{\mathbf{V}\left[\operatorname{Re}\left(H_{i}^{*} \hat{\xi}\right)\right]} .
$$

Then the extrinsic information $L_{D E T}^{e}\left(s_{i}\right)$ of each bit $s_{i}$ detected by the DET is sorted in the required order for creating the sequence $L_{D E T}^{e}\left(\hat{c}_{k, n}^{2}\right)$. This is used as a priori information to be forwarded to the DECs, which computes the more reliable extrinsic information $L_{D E C_{1}}^{e}\left(c_{k}^{1}\right)$ for the next iteration. The iterations are terminated, when a predefined termination criterion is satisfied.

\section{IDM-STC IN COOPERATIVE COMMUNICATIONS}

\section{A. Phase-I Cooperation}

Before transmitting the cooperatively combined IDM-STC signals, all cooperating MSs' information should be exchanged, which we refer to as Phase-I cooperation. We assume the employment of a Time Division Duplexing (TDD) system, where this information is exchanged using different time-slots.

Let us now elaborate further on the Phase-I cooperation scheme of Fig. 3 designed for the IDM-STC arrangement. In time-slot $k$, MS $k$ transmits a sigma mapped symbol $d_{k}=\sum_{n=1}^{N} \hat{c}_{k, n}^{2}$ to all the cooperating MSs. Then the $n$th cooperating receive antenna detects $d_{k}$, extracts $\hat{c}_{k, n}^{2}$ and ignores $\hat{c}_{k, i}^{2}, \forall i \neq n$. Since $N \leq 1 / R$, we have a sufficiently high degree of freedom for detecting each bit of $d_{k}$ using the turbo receiver introduced in Fig. 2. After successfully exchanging information across all the $K \mathrm{MSs}$, the $n$th antenna transmits $\hat{c}_{k, n}^{2}$ of all the $K$ cooperating MSs of Fig. 1.

Thus, the initial Phase-I cooperation of IDM-STC imposes a $K$-slot transmission overhead, which is equal to the number of cooperating MSs $K$, upon exchanging information amongst the cooperating MSs. Hence, as far as the entire TDD system is concerned, setting aside $K$ time-slots for Phase-I cooperation may be viewed as reducing the effective throughput by $K$ time-slots for the sake of achieving $N$ th-order diversity.

\section{B. Effective Throughput}

1) Traditional STBC: Consider having $K=2$ or $K=4$ cooperating MSs in a cluster using a traditional $G_{2}$ STBC [2]:

$$
\mathbf{G}_{2}=\left[\begin{array}{cc}
-x_{2}^{*} & x_{1} \\
x_{1}^{*} & x_{2}
\end{array}\right]
$$

or $G_{4}$ type STBC [10]:

$$
\mathbf{G}_{4}=\left[\begin{array}{cccccccc}
-x_{4}^{*} & -x_{3}^{*} & -x_{2}^{*} & x_{1}^{*} & -x_{4} & -x_{3} & -x_{2} & x_{1} \\
-x_{3}^{*} & x_{4}^{*} & x_{1}^{*} & x_{2}^{*} & -x_{3} & x_{4} & x_{1} & x_{2} \\
x_{2}^{*} & x_{1}^{*} & -x_{4}^{*} & x_{3}^{*} & x_{2} & x_{1} & -x_{4} & x_{3} \\
x_{1}^{*} & -x_{2}^{*} & x_{3}^{*} & x_{4}^{*} & x_{1} & -x_{2} & x_{3} & x_{4}
\end{array}\right] .
$$

The traditional STBC used in cooperative communications operates as follows: 1) the cooperating MSs first exchange their information, which requires $K$ time-slots; 2) given that all MSs now have the signals of all other MSs, conventional STBC transmission of $x_{k}, \forall k$ takes place using all $N=K$ antennas of the $K$ MSs according to the above matrices.

For the sake of achieving a high throughput, each symbol $x_{k}$ can be modulated to an $\mathcal{M}$-ary modulation constellation, where we have $\mathcal{M}=2^{m}$ and $m$ denotes the number of bits/symbol. The effective throughput per user excluding the overhead of the Phase-I inter-MS data exchange can thus be defined as:

$$
\eta_{S T B C}=\frac{r_{S T B C} \times \log _{2} \mathcal{M}}{K} .
$$

2) IDM-STC: In the IDM-STC scheme considered, the overhead imposed by the Phase-I cooperation is constitute by $K$ slots, which is equivalent to the traditional STBC. The effective throughput per user, excluding the overhead of IDM-STC may be expressed as:

$$
\eta_{I D M}=\frac{r_{I D M} \times \mathcal{L}}{K},
$$

where $r_{I D M}$ was defined in Eq. (1) and the number of layers $\mathcal{L}$ was defined in Eq. (2).

\section{Interleaver Allocation}

In our IDM-STC based cooperative scheme, we employ the socalled embedded interleavers of [13], where the $k$ th MS's userspecific interleaver $\pi_{k}^{u}$ constitutes a further interleaved version of the $(k-1)$ st MS's interleaver $\pi_{k-1}^{u}$ using a common "base" interleaver $\pi^{u}$, hence their relationship may be expressed as $\pi_{k}^{u}=\pi^{u}\left(\pi_{k-1}^{u}\right)$. This is the same for the antenna-specific interleaver allocation, where we have $\pi_{n}^{a}=\pi^{a}\left(\pi_{n-1}^{a}\right)$ and $\pi^{a}$ is the common "base" interleaver. These two "base" interleavers are generated randomly in this paper.

\section{BENEFITS OF IDM-STC}

In this section, we compare our IDM-STC based cooperative communications scheme to the traditional $G_{2}$ and $G_{4}$ STBC scheme [10]. We assume that the channels between the $N=K$ cooperating MS transmitters and the BS receiver are i.i.d narrowband Rayleigh fading channels and perfect CSI at the BS receiver. Two scenarios are investigated, namely 1) Fast fading: the channels exhibit a normalized Doppler frequency of $f_{d}=0.02$. 2) Block fading: the channels remain constant over each block but change between different blocks.

We stipulate furthermore that the information exchange amongst MSs is error-free. The transmission frame length in our simulations was set to 800 bits and the maximum number of iterations used was $I=20$. 


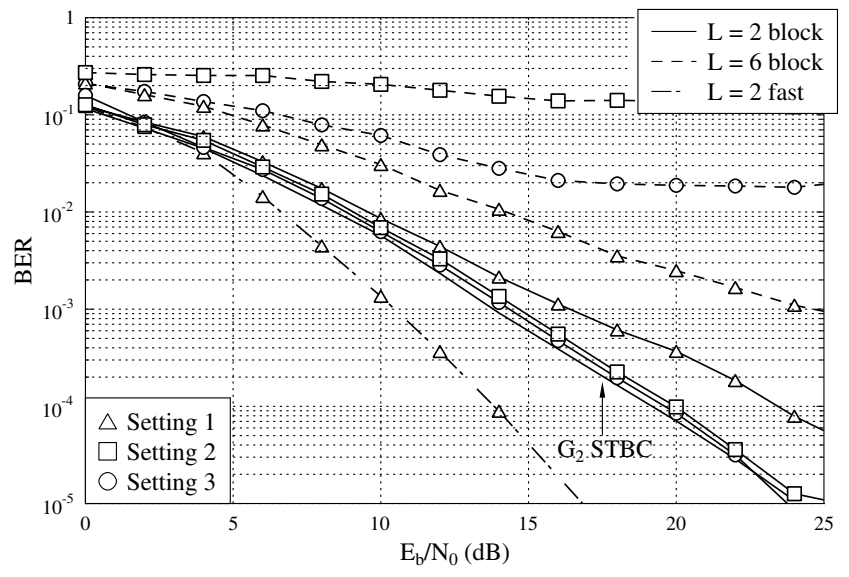

Fig. 4. Comparison of the three typical code-rate settings of IDM-STC to that of $G_{2}$ STBC in cooperative communications.

\section{A. Investigation of IDM-STC}

In this subsection, we illustrate IDM-STC having three typical values of $C^{1}$ and $C^{2}$ as mentioned in Section II, given a fixed total code-rate of $R=1 / 4$ compared to the $G_{2}$ STBC benchmarker represented by the solid line seen in Fig 4, where we have $K=N=$ 2. The three code-rate settings are 1$) r_{1}=1 / 4$ and $\left.r_{2}=1 ; 2\right) r_{1}=1$ and $\left.r_{2}=1 / 4 ; 3\right) r_{1}=1 / 2$ and $r_{2}=1 / 2$.

As seen in Fig 4, in the block-fading scenario, both Setting 2 and 3 have a similar BER as the $G_{2}$ STBC, implying that these two IDM-STCs are capable of achieving full transmit diversity, as the $G_{2}$ STBC. By contrast, Setting 1 has the worst performance, since it is unable to guarantee that the coded bits equally allocated to the transmit antennas, because they may be mapped by the S/P to the same antenna, resulting in a loss of transmit diversity gain. However, when fast fading is encountered, Setting 1 is superior in comparison to the $G_{2}$ STBC, since both transmit diversity and time diversity are available.

When a sufficiently high number of layers, such as $\mathcal{L}=6$ is employed, Setting 1 was seen to be best in Fig 4, while Setting 2 is incapable of supporting the high throughput of Setting 1, which was 3 bits/symbol. This implies that Setting 1 is a highthroughput multiplexing-oriented configuration, while Setting 2 is a low-throughput diversity-oriented configuration. In our forthcoming simulations, we employ Setting 1 aiming at achieving a high rate.

\section{B. Benefit 1 - Power Efficiency}

In this subsection, we consider uniform power allocation.

1) Comparison to $G_{2} S T B C$ : Fig. 5 shows the BER performance of the $G_{2}$ STBC scheme using various $M$-ary modulation schemes and IDM-STC invoking a total code-rate of $R=1 / 4$ and having different number of layers. For fast fading, a single-MS, single-layer system having $N=2$ distributed antennas using IDM-STC was simulated, which served as a benchmarker. Clearly, Fig. 5 suggests that the IDM-STC had a steeper BER slope, when the number of layers $\mathcal{L}$ was as high as 6 and a significant $E_{b} / N_{0}$ gain was observed at $B E R \leq 10^{-4}$. In this case, the effective throughput of IDM-STC was $\eta_{I D M}=6 / 4$ according to Eq. (15), which is equivalent to $G_{2}$ STBC using 8PSK modulation. However, the effective throughput of IDM-STC will be lower than that of a $G_{2}$ STBC using a 4 bits/symbol modulation scheme. By contrast, in the block fading scenario characterized in Fig 5, the IDM-STC is inferior to $G_{2}$ STBC, when the number of IDM-STC layers obeys $\mathcal{L} \geq 4$.

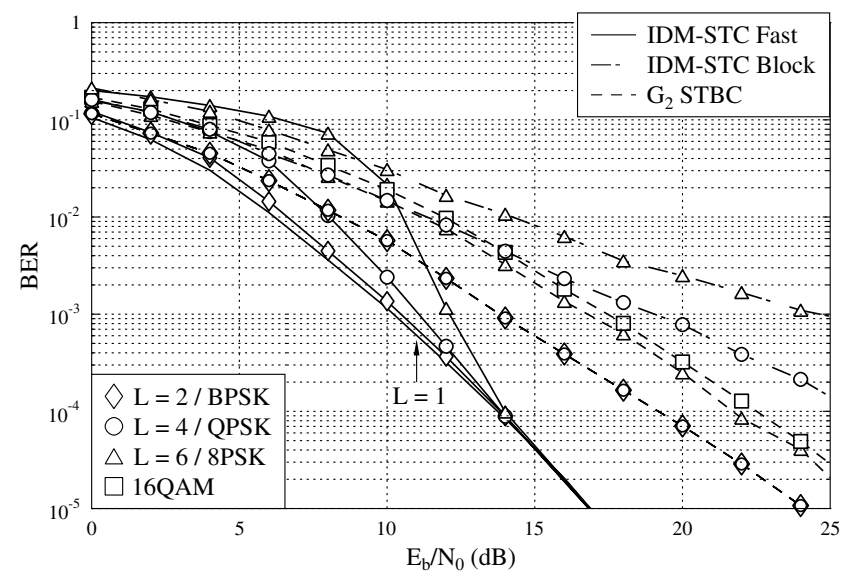

Fig. 5. BER performance of $G_{2}$ STBC and IDM-STC having $R=1 / 4$ over both fast fading and block fading in cooperative communications.

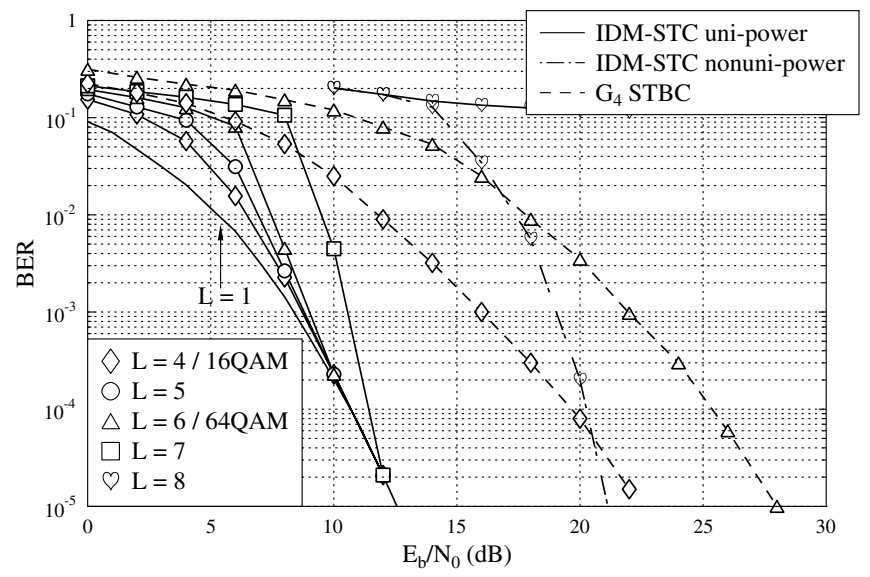

Fig. 6. Performance of $G_{4}$ STBC and IDM-STC over fast fading in cooperative communications, where $\mathcal{L}=4,5,6,7$ layers corresponding upto $7 \mathrm{bits} / \mathrm{symbol}$ transmission were supported. Additionally, with the aid of nonuniform power allocation, $\mathcal{L}=8$ layers can be supported.

2) Comparison to $G_{4} S T B C$ : Let us now compare IDM-STC having $R=1 / 8$ to the above $G_{4}$ STBC in cooperative communications, where we have $K=N=4$. Fig. 7 shows the achievable BER performance of IDM-STC in the block-fading scenario. Although a reduced performance was observed for IDM-STC compared to that of the fast-fading scenario of Fig. 6 owing to the lack of time diversity, they are both superior to that of the $G_{4}$ STBC. In Fig. 6, a single MS assisted by $N=4$ distributed antennas using IDM-STC was characterized, which served as a benchmarker. It can be seen in both Fig. 7 and Fig. 6 that the maximum number of layers $\mathcal{L}$ supported was $\mathcal{L}=7$, which is equivalent to a $G_{4}$ STBC scheme using a large and hence error-sensitive 128-QAM constellation, while requiring a lower power than the $4 \mathrm{bit} / \mathrm{symbol} G_{4}$ STBC aided 16-QAM scheme, as observed at $B E R \leq 10^{-5}$

Thus a significant power gain can be observed compared to the $G_{4}$ STBC both under fast-fading as well as block-fading conditions. In this case we have $r_{I D M}=r_{S T B C}=1 / 2$, implying that IDM-STC does not suffer a rate loss in comparison to $G_{4}$ STBC.

\section{Benefit 2 - Achieving an Increased Throughput}

In this subsection, in addition to uniform phase rotation, nonuniform power allocation is also considered. In this paper, no attempts were made to formally optimize the power allocation scheme. Instead, the following simple non-uniform power allocation strategy [14] 


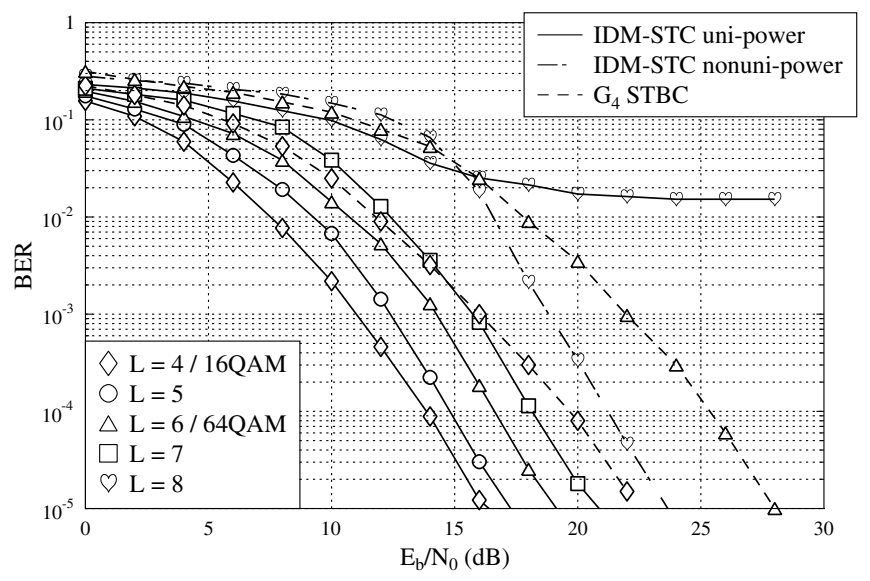

Fig. 7. Performance of $G_{4}$ STBC and IDM-STC over block fading in cooperative communications, where $\mathcal{L}=4,5,6,7$ layers corresponding upto 7 bits/symbol transmission were supported. Additionally, with the aid of nonuniform power allocation, $\mathcal{L}=8$ layers can be supported.

\begin{tabular}{|c|c|c|c|}
\hline$\eta$ & $G_{4} /$ IDM-STC & $\Delta_{1}^{f} / \Delta_{1}^{b}$ & $\Delta_{2}^{f} / \Delta_{2}^{b}$ \\
\hline $4 / 8$ & $\mathcal{M}=16 / \mathcal{L}=4$ & $9 / 6$ & $-/-$ \\
\hline $5 / 8$ & $\mathcal{M}=32 / \mathcal{L}=5$ & $9 / 5$ & $-/-$ \\
\hline $6 / 8$ & $\mathcal{M}=64 / \mathcal{L}=6$ & $14 / 4$ & $24 / 9$ \\
\hline $7 / 8$ & $\mathcal{M}=128 / \mathcal{L}=7$ & $8 / 2$ & $23 / 7$ \\
\hline $8 / 8$ & $\mathcal{M}=256 / \mathcal{L}=8$ & $-1 /-1$ & $4 / 4$ \\
\hline
\end{tabular}

TABLE I

THE POWER GAIN $\Delta$ IN DB OF IDM-STC COMPARED TO $G_{4}$ STBC IN COOPERATIVE COMMUNICATIONS, WHERE $\Delta_{1}, \Delta_{2}$ DENOTES THE POWER GAIN CORRESPONDING TO 16-QAM AND 64-QAM IN $G_{4}$ STBC, RESPECTIVELY. THE SUPERSCRIPT $f$ AND $b$ DENOTE FAST FADING AND BLOCK FADING, RESPECTIVELY.

was adopted ${ }^{4}$. Consider the length- $\mathcal{L}$ weighting coefficient vector $\mathbf{u}$, which obeys:

$$
\rho_{m+1}^{2}=\rho_{m}^{2} / \beta
$$

while ensuring that $\sum_{m=1}^{\mathcal{L}} \rho_{m}^{2}=P_{n}$, where we refer to $\beta \geq 1$ as the scaling factor and $P_{n}$ is the maximum total power of the $n$th transmitter antenna, which is assumed to be equal for all $N$ different antennas.

Returning briefly to Fig. 6 and Fig. 7, they also show the achievable BER performance of IDM-STC, when using non-uniform power allocation. Upon investigating the most appropriate values of $\beta$ experimentally, $\beta=1.2$ was found to be adequate and the number of layers for which an adequate BER performance was attainable was found to be as high as $\mathcal{L}=8$, corresponding to a 256-QAM modulated 8 bits/symbol $G_{4}$ STBC scheme, while requiring a lower power than the 6 bits/symbol $G_{4}$ STBC aided 64-QAM scheme.

The achievable power gain of IDM-STC used in cooperative communications was summarized in Table I, where $\Delta$ was the $E_{b} / N_{0}$ gain of IDM-STC at $B E R=10^{-4}$ over conventional $G_{4}$ STBC scheme having identical-throughput, i.e. we had $\Delta=$ $\left(E_{b} / N_{0}\right)_{S T B C}-\left(E_{b} / N_{0}\right)_{I D M}$.

\section{Benefit 3 - Flexibility}

The design flexibility of IDM-STC allows the employment of an arbitrary number of antennas. This implies that IDM-STC based

\footnotetext{
${ }^{4}$ This power allocation strategy under consideration was derived in the context of capacity region assuming capacity-achieving codes.
}

cooperative communications can be used in diverse cooperative scenarios. More explicitly, when $R=1 / 8$ IDM-STC was used, the system was capable of supporting $K=2,3,4,5$ cooperative MSs without designing different matrices when the traditional STBC code was employed.

Suffice to say, however that in most practical scenarios having a diversity order of more than five attains a near-AWGN BER performance and hence there is limited benefit in further increasing the diversity order, i.e. the number of cooperating antennas, in particular, when considering the effective throughput reduction imposed by the Phase-I inter-MS cooperation. This flexibility is beneficial in terms of forming a flexible cluster of cooperating MSs, allowing MSs to freely join or disjoin the cluster of cooperation.

\section{CONCLUSION}

In this paper, we analyzed the achievable IDM-STC performance in cooperative communications. The Phase-I inter-MS data-exchange was designed and an efficient interleaver allocation scheme was suggested. Compared to the traditional STBC based cooperative design, our proposed system is power-efficient and is capable of achieving a high throughput, especially in the case of non-uniform power allocation. Our scheme is flexible in terms of forming a cluster of cooperating MSs.

\section{REFERENCES}

[1] L. Hanzo, J. Blogh, and S. Ni, 3G, HSDPA, HSUPA and Intelligent FDD versus TDD Networking: Smart Antennas and Adaptive Modulation. IEEE Press - John Wiley, 2007.

[2] S. Alamouti, "A simple transmit diversity technique for wireless communications," IEEE Journal on Selected Areas in Communication, vol. 16, pp. 1451-1458, Oct. 1998.

[3] G. Foschini and M. Gans, "On limits of wireless communications in a fading environment when using multiple antennas," Wireless Personal Communications, vol. 6, pp. 311-355, Mar. 1998.

[4] A. Sendonaris, E. Erkip, and B. Aazhang, "User cooperation diversity. Part I. System description," IEEE Transactions on Communications, vol. 51, pp. 1927-1938, Nov. 2003.

[5] — - "User cooperation diversity. Part II. Implementation aspects and performance analysis," IEEE Transactions on Communications, vol. 51, pp. 1939-1948, Nov. 2003.

[6] A. Stefanov and E. Erkip, "Cooperative space-time coding for wireless networks," IEEE Transactions on Communications, vol. 53, pp. 18041809, Nov. 2005.

[7] K. Wu and L. Ping, "Multilayer turbo space-time codes," IEEE Communications Letters, vol. 9, pp. 55-57, Jan. 2005.

[8] C. Li, K. Li, X. Wang, and L. Ping, "An interleave-division-multiplexing MISO system with partial CSI at transmitter," IEEE Transactions on Vehicular Technology, vol. 56, pp. 1197-1208, May 2007.

[9] L. Hanzo, S. X. Ng, T. Keller, and W. T. Webb, Quadrature Amplitude Modulation: From Basics to Adaptive Trellis-Coded, Turbo-Equalised and Space-Time Coded OFDM, CDMA and MC-CDMA Systems. WileyIEEE Press, 2004.

[10] L. Hanzo, T. Liew, and B. Yeap, Turbo Coding, Turbo Equalisation and Space-Time Coding for Transmission over Fading Channels. WileyIEEE Press, 2002.

[11] X. Ma and L. Ping, "Coded modulation using superimposed binary codes," IEEE Transactions on Information Theory, vol. 50, pp. 33313343, Dec. 2004.

[12] L. Ping, L. Liu, K. Wu, and L. W. K., "Interleave-division multipleaccess," IEEE Transactions on Wireless Communications, vol. 5, pp. 938-947, Apr. 2006.

[13] I. Pupeza, A. Kavcic, and L. Ping, "Efficient generation of interleavers for IDMA," in Proc. of IEEE ICC 06', Istanbul, Turkey, June 11-15, 2006.

[14] P. A. Hoeher and H. Schoeneich, "Interleave-division multiple access from a multiuser point of view," in Proc. 5th Int. Symposium on Turbo Codes and Related Topics in connection with the 6th Int. ITG-Conference on Source and Channel Coding, Munich, Germany, Apr.3-7, 2006, pp. $140-144$ 